\section{Commentary: The future for adult Fontan patients: What is there to see in the liver?}

Kathryn Restaino, $\mathrm{MD},{ }^{\mathrm{a}}$ and
Katsuhide Maeda, $\mathrm{MD}, \mathrm{PhD}^{\mathrm{b}}$

Whereas single ventricle palliation has allowed many infants to survive to adulthood with various univentricular physiologies, the last stage of palliation, Fontan physiology, inevitably results in chronic elevated central venous pressures and relatively low cardiac output. ${ }^{1}$ As a result, end organs such as the liver sustain supraphysiologic venous pressures resulting in chronic congestion. It ultimately leads to fibrosis, cirrhosis, dysfunction, and increased risk for hepatocellular carcinoma. $^{1,2}$

Monitoring and assessment of liver status have become imperative in this population; however, the best way to monitor for irreversible or reversible liver damage is not known. Even the gold standard, biopsy, is challenged by its invasive nature and questioned by patchy disease, sample error, and interreader variability. Noninvasive imaging and scoring systems have not been validated yet in this population. ${ }^{1}$ Shin and colleagues ${ }^{3}$ highlight the poor reliability of traditional noninvasive measures (eg, transient elastography and enhanced liver fibrosis score) of assessing hepatic fibrosis in Fontan patients. We are really puzzled.

The reason liver assessment is so important in these patients is the significant influence it has on future therapy. Patients with Fontan circulatory failure ultimately require consideration of advanced cardiac therapies, including

\footnotetext{
From the ${ }^{\mathrm{a} D i v i s i o n}$ of Cardiology, Department of Pediatrics, and ${ }^{\mathrm{b}}$ Division of Cardiothoracic Surgery, Department of Surgery, Children's Hospital of Philadelphia, Philadelphia, Pa.

Disclosures: The authors reported no conflicts of interest.

The Journal policy requires editors and reviewers to disclose conflicts of interest and to decline handling or reviewing manuscripts for which they may have a conflict of interest. The editors and reviewers of this article have no conflicts of interest.

Received for publication Jan 14, 2022; revisions received Jan 14, 2022; accepted for publication Jan 18, 2022; available ahead of print Jan 22, 2022.

Address for reprints: Katsuhide Maeda, MD, PhD, Division of Cardiothoracic Surgery, Department of Surgery, Children's Hospital of Philadelphia Philadelphia, PA 19104 (E-mail: maedak@chop.edu).

J Thorac Cardiovasc Surg 2022;164:1186-7

$0022-5223 / \$ 36.00$

Copyright (c) 2022 by The American Association for Thoracic Surgery

https://doi.org/10.1016/j.jtcvs.2022.01.023
}

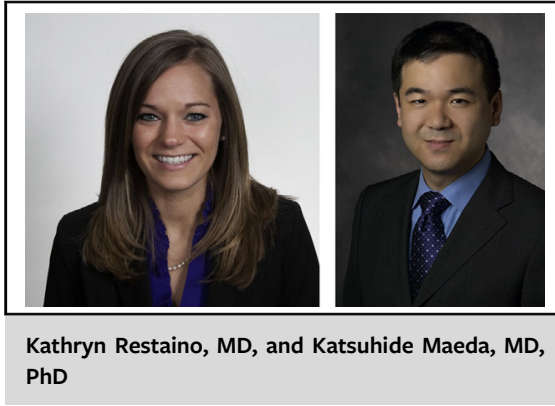

CENTRAL MESSAGE

Liver assessment in Fontan patients has a significant influence on future therapy. Reliable monitoring and assessment modalities are awaited.

ventricular assist devices or/and heart transplant (HT). The important question is the timing of intervention and whether or not HT alone is adequate or if other organs need to be replaced.

For patients who undergo and survive HT, prior studies demonstrate nonprogression of Fontan-associated liver disease in the short and medium term, although longterm risk has not been explored. ${ }^{4}$ Therefore, HT alone seems to be a reasonable option for patients with early disease. However, it is important to recognize the risk that cirrhotic liver disease confers during the immediate peritransplant and posttransplant periods. ${ }^{5}$ This necessitates an accurate diagnosis of cirrhotic liver disease. The presence of synthetic dysfunction, portal hypertension, or other clinical markers have been used; however, there is no consensus on how to determine the need for combined heart-liver transplant. ${ }^{5}$

In fact, it is imperative to refer Fontan patients for advanced heart failure therapies before the progression of liver disease renders them high-risk transplant candidates or liver transplant is required. The need for combined heart-liver transplant contributes to waitlist mortality because donor availability is limited. Future areas of research should include more accurate screening modalities to identify early signs of advanced liver disease. With early and accurate identification of failing physiology, it may be possible to support these patients with ventricular assist devices to promote recovery of liver disease and listing for a single organ transplant. 
We commend the authors on the challenging nature of this study. They provide important insight into the need to investigate alternative noninvasive methods of screening patients with single ventricle physiology for evidence of end organ compromise. With the growing number of adult Fontan survivors-all of whom are affected by some degree of hepatic fibrosis ${ }^{2}$ - validated, noninvasive testing will provide invaluable data.

\section{References}

1. Gordon-Walker TT, Bove K, Veldtman G. Fontan-associated liver disease: a review. J Cardiol. 2019;74:223-32.
2. Goldberg DJ, Surrey LF, Glatz AC, Dodds K, O’Byrne ML, Lin HC, et al. Hepatic fibrosis is universal following Fontan operation, and severity is associated with time from surgery: a liver biopsy and hemodynamic study. J Am Heart Assoc. 2017;6:e04809.

3. Shin YR, Kim SU, Lee S, Choi JY, Park HK, Yoo JE, et al. Noninvasive surrogates are poor predictors of liver fibrosis in patients with Fontan circulation. J Thorac Cardiovasc Surg. 2022;164:1176-85.

4. Broda CR, Alonso-Gonzalez R, Ghanekar A, Gulamhusein A, McDonald M, Luk A, et al. Fate of the liver in the survivors of adult heart transplant for a failing Fontan circulation. J Heart Lung Transplant. 2022;41:283-6. https://doi.org/10. 1016/j.healun.2021.10.020

5. Sganga D, Hollander SA, Vaikunth S, Haeffele C, Bensen R, Navaratnam M, et al. Comparison of combined heart-liver vs heart-only transplantation in pediatric and young adult Fontan recipients. J Heart Lung Transplant. 2021;40: 298-306. 